\title{
Factors Influencing Clinically Meaningful Recanalization after IV-rtPA in Acute Ischemic Stroke
}

A. Murphy, S.P. Symons, J. Hopyan, and R.I. Aviv

\begin{abstract}
BACKGROUND AND PURPOSE: Recanalization may not result in better clinical outcomes after ischemic stroke. We determined the incidence and significant predictors of CMR, defined as CT angiographic recanalization and a good clinical outcome, after IV-rtPA in acute ischemic stroke. A CMR score was devised and tested.
\end{abstract}

MATERIALS AND METHODS: One hundred twenty-six consecutive patients with anterior circulation ischemic stroke receiving IV-rtPA were retrospectively reviewed. Imaging included a baseline NCCT and CTA. Recanalization was assessed on a 24-hour CTA. Clinical outcome was determined by the 90-day mRS. CMR was defined as CTA recanalization and a good clinical outcome (mRS $\leq 2$ ). Logistic regression analysis determined predictors of CMR. The predictive ability of a CMR score was tested with AIC.

RESULTS: CMR occurred in 29\% (36/126). Patients with CMR had fewer neurologic deficits $(P=.001)$ and higher ASPECTS $(P=.041)$ at baseline than those without CMR. Baseline NIHSS score did not predict proximal occlusion (OR 0.959; 95\% $\mathrm{Cl}[0.907-1.014]$; $P=.141$ ). Multivariate analysis showed admission NIHSS score $(P=.001)$ and the site of vessel occlusion $(P=.022)$ to be significant CMR predictors. CMR was significantly less likely in patients with proximal occlusions (ICA, $P=.005$; proximal $\mathrm{M1}, P=.021)$. A CMR score better predicted CMR than either NIHSS or vessel occlusion site alone $(P<.0001)$.

CONCLUSIONS: Milder baseline stroke deficit and distal vessel occlusion are significant predictors of CMR. A combination of these parameters better predicts CMR than either parameter alone.

ABBREVIATIONS: AIC = Akaike information criteria; ASPECTS = Alberta Stroke Program Early CT score; CBS = clot-burden score; CMR = clinically meaningful recanalization; $I A$ = intra-arterial; $I Q R=$ interquartile range; $M I P=$ maximum intensity projection

$T^{1}$ he goal of thrombolytic treatment in acute ischemic stroke is to augment vessel recanalization and to restore blood flow to ischemic but potentially viable tissue. Recanalization is an important end point in several large clinical trials investigating the efficacy of IV and IA thrombolytic treatments. ${ }^{1-3}$ There have been several advances in stroke treatment focused on rapid and more effective restoration of blood flow. ${ }^{4,5}$ Combined protocols utilizing endovascular treatments after initial IV-rtPA are being studied in an attempt to augment vessel patency and improve outcome. $^{4}$

Although recanalization is a beneficial and necessary target in

Received January 17, 2012; accepted after revision March 30.

From the Departments of Medical Imaging (A.M., S.P.S., R.I.V.) and Neurology (J.H.), University of Toronto, Toronto, Ontario, Canada; and Sunnybrook Health Sciences Centre (S.P.S., J.H., R.I.V.), Toronto, Ontario, Canada.

Previously presented in part at: Annual Meeting of the American Society for Neuroradiology, June 4-9, 2011; Seattle, Washington.

Please address correspondence to Amanda Murphy, 211-380 Macpherson Ave, Toronto ON, M4V 3E3, Canada; e-mail: amanda.murphy@live.ca

http://dx.doi.org/10.3174/ajnr.A3169 the treatment of acute stroke, it is not always associated with improved clinical outcome. ${ }^{6-8}$ In the PROACT II and IMS II trials, $26 \%$ and $55 \%$ of patients with recanalization did not show clinical improvement. ${ }^{2}$ Furthermore, both IV and IA therapies for acute stroke are associated with risks that may influence the decision to treat if a good outcome is considered unlikely. ${ }^{4,8-10}$ A recent editorial highlighted that, in the absence of proper patient selection for recanalization, increasing rates of angiographic recanalization are unlikely to result in better clinical outcomes. ${ }^{11}$

Previous investigations have suggested that futile recanalization, or angiographic recanalization in the absence of improved clinical outcome, may be more common in older patients and patients with more severe neurologic deficits. ${ }^{7}$ Other factors such as the size of baseline infarct, location of vessel occlusion, hemorrhagic transformation, and collateral integrity may also influence clinical outcome or recanalization. ${ }^{12}$ Prior studies have evaluated angiographic recanalization or clinical status as independent primary outcomes in the setting of IV-rtPA. However, few have combined CT imaging with clinical outcomes to report CMR, a comprehensive primary end point defined as angiographic recan- 
alization and a good clinical outcome. ${ }^{7,12-16}$ We analyzed clinical and CT imaging data from an acute ischemic stroke data base to determine the incidence of, and the factors contributing to, CMR.

\section{MATERIALS AND METHODS Study Design}

An analysis was performed of consecutive acute anterior circulation ischemic stroke admissions to a tertiary stroke center between January 2007 and January 2010. All subjects presented $<3$ hours after the onset of symptoms, demonstrated a vessel occlusion on baseline CTA, and were treated only with IV-rtPA (0.9 $\mathrm{mg} / \mathrm{kg}$ ). All patients underwent a standard stroke imaging protocol, including a baseline NCCT and CTA, a 24-hour CTA, and a 5to 7-day MRI $(n=126)$. Clinical and radiologic data were collected by a stroke neurologist (5 years of experience) and neuroradiologist (6 years of experience). All procedures were in accordance with institutional guidelines and were approved by the institutional review board. All subjects or substitute decision makers provided informed consent for the use of clinical and radiologic data.

\section{Clinical Parameters}

Baseline data collected for all patients included NIHSS score, time from symptom onset to presentation, blood glucose, blood pressure, time to IV-rtPA, and rtPA dose. Cardiovascular risk factors, including history of hypertension, coronary artery disease, atrial fibrillation, hypercholesterolemia, smoking, hypertension, and diabetes, were recorded. Clinical outcome was assessed using the $\mathrm{mRS}$ at 90 days. A good clinical outcome was defined by $\mathrm{mRS} \leq 2$.

\section{Radiologic Parameters}

An experienced neuroradiologist (6 years of experience), blinded to clinical data including final clinical outcome, collected all radiologic data. Baseline ASPECTS, collateral score, CBS, and location of vessel occlusion were noted for each patient at admission. Briefly, ASPECTS assigns a 10-point score to the affected hemisphere; 1 point is subtracted for each region of NCCT hypoattenuation. All patients in this study were eligible for IV-rtPA therapy and therefore did not have baseline ASPECTS $<5$ or hypoattenuation > one-third of the MCA territory. CBS assigns 10 points to normal ipsilateral vasculature and subtracts points depending on clot location and distribution. ${ }^{17,18}$ Two points each are deducted for the supraclinoid ICA segment, proximal MCA, and distal MCA. Infraclinoid ICA, anterior cerebral artery, and the M2 segment of the MCA are each assigned 1 point.

CT angiographic recanalization was assessed by comparing baseline to follow-up CTA axial and multiplanar MIP images on a PACS; CTA recanalization was considered present when complete or partial recanalization of the occluded arterial segment was identified. Proximal vessel occlusions included those within the ICA and/or the proximal half of the M1 MCA segment. Distal occlusions were within and distal to the lateral half of the M1 MCA segment. Coronal CTA MIP images were used to determine the length of the MCA and select a midpoint to divide the vessel into proximal and distal M1 segments. The division of MCA into proximal and distal accounts for the theoretic origin of collateral pathways through the proximal and distal lenticulostriate vessels, respectively. This approach has been recently validated. ${ }^{19}$

Hemorrhagic transformation was assessed with MR imaging at day 5, 6, or 7. The presence of either hemorrhagic infarction or parenchymal hemorrhage was considered positive for hemorrhage. ${ }^{20} \mathrm{CMR}$ was defined as the composite of $\mathrm{CT}$ angiographic recanalization with a good clinical outcome $(\mathrm{mRS} \leq 2)$.

\section{Scan Acquisition Parameters and Scan Protocol}

The CT stroke protocol was performed on a 64-section CT scanner. CTA was performed from the aortic arch to vertex with iodinated contrast agent up to a maximum of $90 \mathrm{~mL}$ injection at 5 $\mathrm{mL} / \mathrm{s}$ with a 5- to 10-second delay. Multiplanar 7-mm MIP reconstructions and 4-mm axial CTA source image reformats were automatically generated at the scanner console and sent to the PACS. MR imaging sequences included axial FLAIR, DWI, T2 FSE, and sagittal T1 FLAIR.

\section{Statistical Analysis}

The mean and standard deviation were calculated for age, admission blood glucose, admission systolic blood pressure, and time to IV-rtPA. Differences between patients with and without CTA recanalization, good clinical outcome, and CMR were determined with a 1-way ANOVA and post hoc Bonferroni analyses. The median and IQR were calculated for the ASPECTS, admission NIHSS score, CBS, and collateral score. Differences between groups were determined using a Kruskal-Wallis nonparametric analysis. Differences in the frequency of clinical risk factors between groups were compared with the Mann-Whitney test.

Occlusion location was subdivided into ICA, proximal M1, distal M1, and M2-M4 segments of the MCA. Differences in occlusion location between patients with and without CTA recanalization, good clinical outcome, and CMR were determined using a Kruskal-Wallis nonparametric univariate analysis. Additional logistic regression analysis was performed to determine the relationship between location of vessel occlusion and CMR. Odds ratios and confidence intervals were calculated for each occlusion location after dichotomization of vessel occlusion into proximal (ICA and proximal M1) and distal (distal M1-M4). The relationship between NIHSS score and proximal occlusions was investigated with logistic regression and receiver operating characteristic analysis. Multivariate logistic regression analysis was used to determine significant predictors of successful recanalization. Age, baseline NIHSS, ASPECTS, CBS, time to IV-rtPA, occlusion location, and hemorrhagic transformation were entered into the model. Based on these results, a CMR score that included both admission NIHSS and occlusion location was devised and tested. NIHSS was divided into 4 categories of severity as previously published: $0=$ NIHSS $0-7 ; 1=$ NIHSS $8-14 ; 2=$ NIHSS 15-20; $3=$ NIHSS $>20 .{ }^{21}$ For occlusion location, proximal occlusions (ICAproximal M1) were scored 1 and distal occlusions (distal M1-M4) were scored 0 . The CMR score was the sum of the stratified NIHSS score plus the occlusion score; the total CMR score for each patient ranged from $0-4$.

The number and percentage of patients achieving CMR were stratified according to the CMR score. Akaike's information criterion $(\mathrm{AIC}=$ likelihood of the model $+2 \times$ number of param- 
Table 1: Baseline clinical data

\begin{tabular}{lc}
\hline ASPECTS (median [IQR]) & $7.0(3.0)$ \\
Admission NIHSS score (median [IQR]) & $15.0(11.0)$ \\
Time to presentation (min) & $116.1(89.1)$ \\
Time to IV-rtPA (min) & $153.2(49.6)$ \\
Total IV-rtPA dose (mg) & $60.4(18.7)$ \\
\hline
\end{tabular}

Note:-Values are mean (standard deviation) unless otherwise specified.

eters) was used to determine which of the significant predictors, either independently or as a combined score, provided the best model for predicting CMR. ${ }^{22}$ The predictive value was also quantified by the entropy, $\mathrm{r}^{2}$, which equals $\left(\mathrm{L}_{\mathrm{O}}-\mathrm{L}_{\mathrm{M}}\right) / \mathrm{L}_{\mathrm{O}}$, where $\mathrm{L}_{\mathrm{O}}$ and $\mathrm{L}_{\mathrm{M}}$ represent the maximized -2 (log likelihood) of the null model and the fitted model, respectively. $P<.05$ was considered significant.

\section{RESULTS}

\section{Frequency of Recanalization}

Summary data for the study population are shown in Table 1 . The mean age was $72.8 \pm 12.1$ years (men $64 / 126,51 \%$ ). CT angiographic recanalization occurred in 58\% (73/126), good outcome was observed in 36\% (45/126), and CMR occurred in 29\% (36/ 126) of all patients. Of 73 patients with CT angiographic recanalization, 48\% (35/73) had a good outcome (mRS $\leq 2)$.

\section{Comparison of Baseline Parameters for CT Angiographic Recanalization, Good Outcome, and Clinically Meaningful Recanalization}

Tables 2 and 3 show the results of the univariate analysis in each of the 3 groups of patients, with and without recanalization, good outcome, and CMR. Patients with CMR had fewer neurologic deficits $(P<.001)$ and higher ASPECTS $(P=.041)$ than those without CMR. In contrast, the ASPECTS and NIHSS scores were not significantly different between patients with and without CTA recanalization. Collateral score was not significantly different between the 3 groups.

\section{Clot Location}

Clot location was significantly different between groups with and without recanalization, good clinical outcome, and CMR. Post hoc analysis showed that CT angiographic recanalization $(P<$ $.0001)$ and CMR $(P=.008)$ were less frequent in the presence of ICA occlusions (Fig 1). The frequency of CMR was significantly less when occlusions were located in the ICA $(8.3 \% ; P<.0001)$ or in the proximal M1 segment of the MCA $(11.1 \% ; P=.001)$. Of 48 patients with a proximal occlusion (ICA or proximal M1), only $14.6 \%$ (7/48) achieved CMR.

The presence of either ICA occlusion (OR 0.201; 95\% CI [0.057-0.712]; $P=.005$ ) or proximal M1 occlusions (OR 0.345; 95\% CI [0.130-0.917]; $P=.021)$ was a significant predictor of not achieving CMR.

Baseline NIHSS score was not a significant predictor of proximal vessel occlusion (OR 0.959; 95\% CI [0.907-1.014]; $P=.141$ ) with an AUC of $0.587(P=.103)$.

\section{Predictors of CMR and Performance of the CMR Score}

Following multivariate analysis, admission NIHSS score $(P=$ $.001)$ and proximal site of vessel occlusion $(P=.022)$ remained significant predictors of CMR. A lower admission NIHSS score
(OR 0.926; 95\% CI [0.865-0.991]; $P=.003$ ) was associated with a higher likelihood of CMR, whereas CMR was less likely with proximal occlusions. Age, ASPECTS, time to IV-rtPA, CBS, and hemorrhagic transformation were not significant predictors of CMR.

The probability of clinically meaningful recanalization decreased with increasing CMR score (Table 4). Whereas 9/13 (69\%) patients with a score of 0 achieved CMR, no patients with a score of 4 demonstrated CMR. The CMR score performed better for predicting clinically meaningful recanalization than either NIHSS or occlusion location alone (AIC 132.2, $R^{2} 13.5 \%$; $P<$ .0001) (Table 5).

\section{DISCUSSION}

We demonstrate that CMR was achieved in $29 \%$ of consecutive patients treated with IV-rtPA for acute anterior circulation stroke. Lower admission NIHSS score and a distal clot location are each significant predictors of CMR; however, a composite CMR score combining NIHSS and clot location is an even stronger predictor of CMR than either parameter alone. Importantly, NIHSS was not significantly associated with proximal occlusions. Proximal occlusions were significantly less likely to be associated with a good clinical outcome than distal vessel occlusions. Only $14.6 \%$ of patients with proximal vessel occlusion achieved CMR.

Indisputably, the goal of thrombolytic therapy is to achieve recanalization and a good clinical outcome or "clinically meaningful recanalization." According to stroke guidelines based on National Institute of Neurological Disorders and Stroke and European Cooperative Acute Stroke Study data, the decision to treat with IV-rtPA depends upon the exclusion of specific clinical and imaging parameters. ${ }^{20,23}$ Imaging exclusions are limited to the presence of hemorrhage and large hypoattenuation on NCCT. ${ }^{24}$ Proximal vessel occlusion is not considered a contraindication to IV-rtPA, despite poorer recanalization rates and outcomes reported by others and demonstrated in this study. ${ }^{12}$ The poor rate of CMR for proximal occlusions demonstrated in this study has clinical ramifications in the many centers where noninvasive vascular imaging is not, or cannot, be performed before thrombolysis treatment. The present data demonstrate that the identification of clot location with vascular imaging may provide crucial information for the purposes of triaging patients to IA versus IV therapy.

Our earlier practice, like many other centers, was to administer IV-rtPA to all eligible patients, irrespective of site of vessel occlusion, in accordance with stroke guidelines. However, our results indicate that patients harboring proximal vessel occlusions have only a $14.6 \%$ chance of achieving CMR after administration of IV-rtPA. Similarly, a recent study found that in IV-rtPAtreated patients, a proximal occlusion was associated with a 2.4 times higher risk of nonrecanalization, while no such association was seen for distal MCA occlusions. ${ }^{19}$ Significantly, a study assessing factors associated with futile recanalization in IA-treated patients found no association with clot location on multivariate analysis. Futile recanalization did, however, occur less frequently with more distal occlusions. ${ }^{7}$ The theoretic reduced time to restoring luminal patency and increased recanalization rates for IA therapy in patients with proximal occlusions may explain why outcome is independent of vessel occlusion site in IA-treated patients. These, and our findings, suggest that IA therapy may be 
Table 2: Univariate analysis in patients with and without recanalization, good outcome, or CMR

\begin{tabular}{|c|c|c|c|c|c|c|}
\hline & \multicolumn{2}{|c|}{ CT Angiographic Recanalization } & \multicolumn{2}{|c|}{ Good Outcome } & \multicolumn{2}{|c|}{ Clinically Meaningful Recanalization } \\
\hline & $\begin{array}{c}\text { Yes } \\
(n=73)\end{array}$ & $\begin{array}{c}\text { No } \\
(n=53)\end{array}$ & $\begin{array}{c}\text { Yes } \\
(n=45)\end{array}$ & $\begin{array}{c}\text { No } \\
(n=81)\end{array}$ & $\begin{array}{c}\text { Yes } \\
(n=36)\end{array}$ & $\begin{array}{c}\text { No } \\
(n=90)\end{array}$ \\
\hline Age (yr) (mean) (SD) & 74.4 (11.9) & $70.6(12.1)$ & $70.0(13.3)$ & $74.4(11.2)$ & 70.5 (13.7) & $73.7(11.4)$ \\
\hline ASPECTS & $8.0(6.0-9.0)$ & $6.0(5.0-9.0)$ & $8.0(6.0-9.0)$ & $7.0(5.0-9.0)^{\mathrm{a}}$ & $8.0(6.25-9.0)$ & $7.0(5.0-9.0)^{\mathrm{a}}$ \\
\hline Hypertension (frequency) (\%) & $45(61.6 \%)$ & $37(69.8 \%)$ & $24(53.3 \%)$ & $58(71.6 \%)^{\mathrm{a}}$ & $20(55.6 \%)$ & $62(68.9 \%)$ \\
\hline Diabetes (frequency) (\%) & $11(15.1 \%)$ & $11(20.8 \%)$ & $5(10.9 \%)$ & $17(21.3 \%)$ & $5(13.9 \%)$ & $17(18.9 \%)$ \\
\hline Hypercholesterolemia (frequency) (\%) & $26(35.6 \%)$ & $17(32.1 \%)$ & $14(30.4 \%)$ & $29(36.3 \%)$ & $13(36.1 \%)$ & $30(33.3 \%)$ \\
\hline Coronary artery disease (frequency) (\%) & $20(27.4 \%)$ & $8(15.1 \%)$ & $11(23.9 \%)$ & $17(21.3 \%)$ & $10(27.8 \%)$ & $18(20.0 \%)$ \\
\hline Atrial fibrillation (frequency) (\%) & $25(34.2 \%)$ & $15(28.3 \%)$ & $16(34.8 \%)$ & $24(30.0 \%)$ & $11(30.6 \%)$ & $29(32.2 \%)$ \\
\hline Smoking (frequency) (\%) & $10(13.7 \%)$ & $13(24.5 \%)$ & $11(23.9 \%)$ & $12(15.0 \%)$ & $8(22.2 \%)$ & $15(16.7 \%)$ \\
\hline NIHSS score & $14.0(9.0-19.5)$ & $17.0(10.0-20.0)$ & $10.0(5.0-17.0)$ & $18.0(12.5-21.0)^{\mathrm{a}}$ & $9.5(5.25-18.0)$ & $17.0(11.75-21.0)^{\mathrm{a}}$ \\
\hline Blood glucose (mg/dL) (mean) (SD) & $7.5(5.9)$ & $7.8(2.5)$ & $6.5(1.3)$ & $8.3(5.8)^{a}$ & $6.4(1.3)$ & $8.2(5.5)$ \\
\hline $\mathrm{SBP}(\mathrm{mm} \mathrm{Hg})\left(\right.$ mean) $(\mathrm{SD})^{\mathrm{b}}$ & $159(25.8)$ & $158(25.8)$ & $159.8(29.7)$ & $157.9(25.9)$ & $161.5(30.6)$ & $157.4(25.9)$ \\
\hline Time to IV-rtPA (min) (mean) (SD) & $157.1(54.4)$ & $147.9(42)$ & $150.4(37.8)$ & $154.8(55.2)$ & $152.4(38.2)$ & $153.6(53.7)$ \\
\hline CBS & $7.0(6.0-9.0)$ & $6.0(3.0-8.5)^{\mathrm{a}}$ & $8.0(6.0-9.0)$ & $6.0(4.0-8.5)^{\mathrm{a}}$ & $7.0(6.0-9.0)$ & $6.0(4.0-9.0)$ \\
\hline Collateral score & $2.0(2.0-3.0)$ & $2.0(1.0-3.0)$ & $2.0(2.0-3.0)$ & $2.0(1.0-3.0)$ & $2.0(2.0-3.0)$ & $2.0(1.0-3.0)$ \\
\hline HT (frequency) (\%) & $73(58)$ & $53(42)$ & $17(38)$ & $28(62)$ & $13(36)$ & $46(51)$ \\
\hline
\end{tabular}

Note:-Unless otherwise noted, data are median (IQR). $P$ values were obtained with ANOVA univariate analysis with post-hoc Bonferroni correction or with Kruskal-Wallis nonparametric analysis. HT indicates hemorrhagic transformation; SBP, systolic blood pressure.

a $P<0.05$ was considered to indicate a significant difference.

${ }^{\mathrm{b}} n=125$ for SBP due to missing data for 1 subject.

Table 3. Clot location in patients with and without recanalization, good outcome, or CMR

\begin{tabular}{|c|c|c|c|c|c|c|}
\hline \multirow[b]{2}{*}{ Clot Location } & \multicolumn{2}{|c|}{$\begin{array}{l}\text { CT Angiographic } \\
\text { Recanalization }\end{array}$} & \multicolumn{2}{|c|}{ Good Outcome } & \multicolumn{2}{|c|}{$\begin{array}{l}\text { Clinically Meaningful } \\
\text { Recanalization }\end{array}$} \\
\hline & $\begin{array}{c}\text { Yes } \\
(n=73)\end{array}$ & $\begin{array}{c}\text { No } \\
(n=53)\end{array}$ & $\begin{array}{c}\text { Yes } \\
(n=45)\end{array}$ & $\begin{array}{c}\text { No } \\
(n=81)\end{array}$ & $\begin{array}{c}\text { Yes } \\
(n=36)\end{array}$ & $\begin{array}{c}\text { No } \\
(n=90)\end{array}$ \\
\hline ICA & $8(11.0)^{a}$ & $23(43.4)$ & $7(15.6)$ & $24(29.6)$ & $3(8.3)^{a}$ & $28(31.1)$ \\
\hline Proximal M1 & $10(13.7)^{a}$ & $7(13.2)$ & $4(8.9)^{a}$ & 13 (16.0) & $4(11.1)^{a}$ & $13(14.4)$ \\
\hline Distal M1 & 27 (37.0) & 10 (18.9) & $13(28.9)$ & $24(29.6)$ & $14(38.9)$ & $23(25.6)$ \\
\hline $\mathrm{M} 2-\mathrm{M} 4$ & $28(38.4)$ & $13(24.5)$ & $21(46.7)^{\mathrm{a}}$ & $20(24.7)$ & $15(41.7)$ & $26(28.9)$ \\
\hline
\end{tabular}

Note:-Location represents the most proximal site of occlusion. Data are frequency (\%). $P$ values were obtained with Kruskal-Wallis nonparametric analysis, followed by Mann-Whitney $U$ post hoc tests.

${ }^{a} P<.05$ was considered to indicate a significant difference.

beneficial in patients with proximal occlusions and potentially avoids the poor outcomes reported for IV-rtPA. ${ }^{4,5}$ With increased focus on clinically meaningful, rather than angiographic, recanalization, ${ }^{11}$ our results underscore the importance of utilizing advanced imaging to triage appropriate patients with proximal occlusions to IA therapy. In the absence of an interventional service, the decision to treat proximal occlusions with IV-rtPA should be made with careful consideration of the potential for exposing patients to more harm than benefit.

That NIHSS score and proximal occlusion site are associated with CMR is intuitive and indeed many clinical and imaging parameters have been shown to be associated with good clinical outcome or angiographic recanalization after stroke thrombolysis. ${ }^{1,7,18,25,26}$ However, very few studies have evaluated both radiologic and clinical factors in the context of CMR. Von Kummer et $\mathrm{al}^{12}$ showed that a good clinical outcome was more likely in the presence of more distal occlusions, good baseline function, and angiographic recanalization within 24 hours of treatment with IA or IV thrombolysis. Hussein et $\mathrm{al}^{7}$ evaluated combined clinical and radiologic factors, but did not include baseline imaging and thus could not evaluate the effect of infarct size or collateral score on outcome. A recent editorial, addressing the issue of futile recanalization, acknowledged that baseline infarct was 1 of 4 key features that need to be considered when evaluating CMR. ${ }^{27} \mathrm{Un}$ like the present study, Hussein et $\mathrm{al}^{7}$ included only patients treated by IA thrombolysis, limiting its applicability to most the acute stroke population. Therefore, further investigation into the factors that are associated with CMR, and not only angiographic recanalization or good clinical outcomes, is needed.

Although a higher NIHSS score may be seen with more proximal occlusions, a recent study of 699 patients demonstrated that $55 \%$ of patients with a proximal occlusion amenable to interventional therapy had an NIHSS score $\leq 10$. The sensitivity for detection of a proximal occlusion in that study was only $48 \%$ using an NIHSS cutoff of $\geq 10 .^{28}$ Similarly, the DIAS-2 phase III study demonstrated high NIHSS in the absence of vessel occlusions in up to $30 \%$ of patients. ${ }^{29}$ Importantly, in the present series, we showed no significant relationship between NIHSS score and site of vessel occlusion. Indeed, the presented multivariate analysis shows the need to consider both the clinical deficit at presentation (scored by the NIHSS) and the site of vessel occlusion when considering IV thrombolytic therapy.

Our findings emphasize the need for pooling of individual series and further studies that incorporate multimodal imaging. These data would provide clinicians with insight into baseline imaging and clinical features associated with CMR and facilitate risk stratification for future treatment. ${ }^{13,11,19}$ Understanding the factors that determine outcome may also guide the physician in counseling of family and patients when route of treatment and the anticipated prognosis for treatment or withholding treatment is discussed. Finally, with in- 

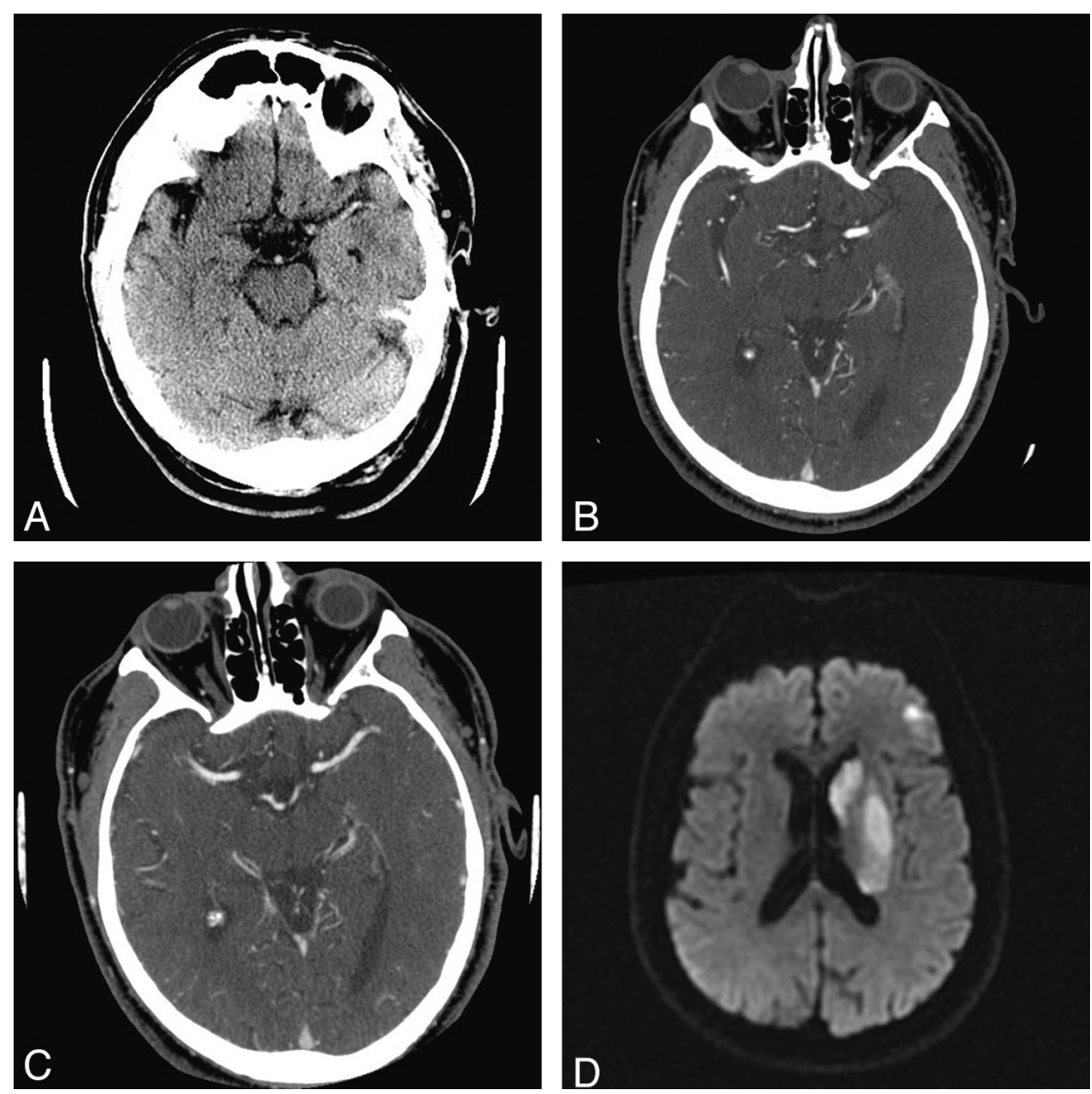

FIG 1. Recanalization of MCA occlusion without a good clinical outcome. A 67-year-old male patient presented 141 minutes after stroke onset. NIHSS score at presentation was 13 , reducing to 10 following $99 \mathrm{mg}$ of IV-rtPA. A, Baseline NCCT demonstrates a hyperattenuated left MI MCA, ASPECTS of 6 (not shown), and CMR score = 2. B, CTA confirmed an MCA occlusion. C, Follow-up CTA demonstrates recanalization, and $(D)$ an infarct within the left basal ganglia and frontal operculum on DWI. The 90-day mRS was 4.

Table 4: Number and percentage of patients achieving a clinically meaningful recanalization stratified against the CMR score

\begin{tabular}{lc}
\hline CMR Score & Frequency of CMR (\%) \\
\hline 0 & $9 / 13(69 \%)$ \\
1 & $12 / 34(35 \%)$ \\
2 & $11 / 35(31 \%)$ \\
3 & $4 / 31(13 \%)$ \\
4 & $0 / 13(0 \%)$ \\
\hline
\end{tabular}

Note:-CMR score is the sum of the stratified NIHSS score and the occlusion location score. For NIHSS, $0=$ NIHSS 0 -7; $1=$ NIHSS 8-14; $2=$ NIHSS 15-20; $3=$ NIHSS $>20$. For occlusions, ICA and proximal $M 1=1$; distal $M 1$ and $M 2-M 4$ segments $=0$. Scores range from $0-4$. A higher CMR corresponded to a lower frequency of successful recanalization with IV-rtPA

\section{Table 5: AIC analysis}

\begin{tabular}{lrrrrr}
\hline & AIC & $\mathbf{R}_{\mathbf{2}}$ & $\boldsymbol{\chi}^{\mathbf{2}}$ (df) & $\boldsymbol{P}$ Value \\
\hline Null model & 150.2 & & & \\
$\begin{array}{l}\text { Fitted model with admission NIHSS } \\
\text { Fitted model with occlusion }\end{array}$ & 136.4 & $10.7 \%$ & 15.8 & $<.0001$ \\
$\quad$ location & 144.7 & $5.1 \%$ & 7.5 & .0062 \\
$\begin{array}{l}\text { Fitted model with both NIHSS and } \\
\quad \text { occlusion location (CMR) }\end{array}$ & 132.12 & $13.5 \%$ & 20.1 & $<.0001$ \\
\hline
\end{tabular}

Note:-Performance of combining stratified NIHSS score and vessel occlusion location (CMR score) versus each alone. The null model was compared with a model fitted with admission NIHSS score, occlusion location, and the CMR score. To quantify the predictive effects of each parameter on CMR, the AIC and coefficient of determination $R^{2}$ were calculated. A better model is evidenced by a lower AIC and a higher $R^{2}$. 
Disclosures: Dr. Murphy received a summer student scholarship from the Heart and Stroke Foundation Centre for Stroke Recovery; Dr. Symons is an advisor to Bayer, unrelated to the present research.

\section{REFERENCES}

1. del Zoppo GJ, Higashida RT, Furlan AJ, et al. PROACT: a phase II randomized trial of recombinant pro-urokinase by direct arterial delivery in acute middle cerebral artery stroke. PROACT investigators. Prolyse in Acute Cerebral Thromboembolism. Stroke 1998;29:4-11

2. Furlan A, Higashida R, Wechsler L, et al. Intra-arterial prourokinase for acute ischemic stroke. The PROACT II study: a randomized controlled trial. Prolyse in Acute Cerebral Thromboembolism. JAMA 1999;282:2003-11

3. Shi ZS, Loh Y, Walker G, et al. Clinical outcomes in middle cerebral artery trunk occlusions versus secondary division occlusions after mechanical thrombectomy: pooled analysis of the Mechanical Embolus Removal in Cerebral Ischemia (MERCI) and multi MERCI trials. Stroke 2010;41:953-60

4. Smith WS. Safety of mechanical thrombectomy and intravenous tissue plasminogen activator in acute ischemic stroke. Results of the Multi Mechanical Embolus Removal in Cerebral Ischemia (MERCI) trial, part I. AJNR Am J Neuroradiol 2006;27:1177-82

5. Menon BK, Hill MD, Eesa M, et al. Initial experience with the Penumbra stroke system for recanalization of large vessel occlusions in acute ischemic stroke. Neuroradiology 2010;53:261-66

6. Smith WS, Sung G, Saver J, et al. Mechanical thrombectomy for acute ischemic stroke: final results of the multi MERCI trial. Stroke 2008;39:1205-12

7. Hussein HM, Georgiadis AL, Vazquez G, et al. Occurrence and predictors of futile recanalization following endovascular treatment among patients with acute ischemic stroke: a multicenter study. AJNR Am J Neuroradiol 2010;31:454-58

8. IMS II Trial Investigators. The Interventional Management of Stroke (IMS) II study. Stroke 2007;38:2127-35

9. Singer OC, Berkefeld J, Lorenz MW, et al. Risk of symptomatic intracerebral hemorrhage in patients treated with intra-arterial thrombolysis. Cerebrovasc Dis 2009;27:368-74

10. Kase CS, Furlan AJ, Wechsler LR, et al. Cerebral hemorrhage after intra-arterial thrombolysis for ischemic stroke: the PROACT II trial. Neurology 2001;57:1603-10

11. Cloft HJ. Death and destruction in the intra-arterial battle with acute ischemic stroke. AJNR Am J Neuroradiol 2011;32:1769-70

12. von Kummer R, Holle R, Rosin L, et al. Does arterial recanalization improve outcome in carotid territory stroke? Stroke 1995;26:581-87

13. Marks MP, Olivot JM, Kemp S, et al. Patients with acute stroke treated with intravenous tPA 3-6 hours after stroke onset: correlations between MR angiography findings and perfusion- and diffusion-weighted imaging in the DEFUSE study. Radiology 2008;249:614-23

14. Khatri P, Abruzzo T, Yeatts SD, et al. Good clinical outcome after ischemic stroke with successful revascularization is time-dependent. Neurology 2009;73:1066-72

15. Zaidat OO, Suarez JI, Santillan C, et al. Response to intra-arterial and combined intravenous and intra-arterial thrombolytic therapy in patients with distal internal carotid artery occlusion. Stroke 2002;33:1821-26

16. Alexandrov AV. Current and future recanalization strategies for acute ischemic stroke. J Intern Med 2010;267:209-19

17. Puetz V, Dzialowski I, Hill MD, et al. Intracranial thrombus extent predicts clinical outcome, final infarct size and hemorrhagic transformation in ischemic stroke: the clot burden score. Int J Stroke 2008;3:230-36

18. Tan IY, Demchuk AM, Hopyan J, et al. CT angiography clot burden score and collateral score: correlation with clinical and radiologic outcomes in acute middle cerebral artery infarct. AJNR Am J Neuroradiol 2009;30:525-31
19. Mendonca N, Rodriguez-Luna D, Rubiera M, et al. Predictors of tissue-type plasminogen activator nonresponders according to location of vessel occlusion. Stroke 2011;43:417-21

20. Hacke W, Kaste M, Fieschi C, et al. Intravenous thrombolysis with recombinant tissue plasminogen activator for acute hemispheric stroke. The European Cooperative Acute Stroke Study (ECASS). JAMA 1995;274:1017-25

21. Adams HP Jr, Leclerc JR, Bluhmki E, et al. Measuring outcomes as a function of baseline severity of ischemic stroke. Cerebrovasc Dis 2004;18:124-29

22. Wagenmakers EJ, Farrell S. AIC model selection using Akaike weights. Psychon Bull Rev 2004;11:192-96

23. The National Institute of Neurological Disorders and Stroke Rt-PA Stroke Study Group. Tissue plasminogen activator for acute ischemic stroke. $N$ Engl J Med 1995;333:1581-87

24. Adams HP Jr, del Zoppo G, Alberts MJ, et al. Guidelines for the early management of adults with ischemic stroke: a guideline from the American Heart Association/American Stroke Association Stroke Council, Clinical Cardiology Council, Cardiovascular Radiology and Intervention Council, and the Atherosclerotic Peripheral Vascular Disease and Quality of Care Outcomes in Research interdisciplinary working groups: the American Academy of Neurology affirms the value of this guideline as an educational tool for neurologists. Stroke 2007;38:1655-711

25. Barber PA, Demchuk AM, Zhang J, et al. Validity and reliability of a quantitative computed tomography score in predicting outcome of hyperacute stroke before thrombolytic therapy. ASPECTS study group. Alberta Stroke Programme Early CT Score. Lancet 2000;355:1670-74

26. Adams HP Jr, Davis PH, Leira EC, et al. Baseline NIH stroke scale score strongly predicts outcome after stroke: a report of the Trial of Org 10172 in Acute Stroke Treatment (TOAST). Neurology 1999;53:126-31

27. Goyal M. Poor clinical outcome despite successful arterial recanalization. What went wrong? How can we do better? Neuroradiology 2010;52:341-43

28. Maas MB, Furie KL, Lev MH, et al. National Institutes of Health Stroke Scale Score is poorly predictive of proximal occlusion in acute cerebral ischemia. Stroke 2009;40:2988-93

29. Hacke W, Furlan AJ, Al-Rawi Y, et al. Intravenous desmoteplase in patients with acute ischaemic stroke selected by MRI perfusiondiffusion weighted imaging or perfusion CT (DIAS-2): a prospective, randomised, double-blind, placebo-controlled study. Lancet Neurol 2009;8:141-50

30. Davis SM, Donnan GA, Parsons MW, et al. Effects of alteplase beyond $3 \mathrm{~h}$ after stroke in the Echoplanar Imaging Thrombolytic Evaluation Trial (EPITHET): a placebo-controlled randomised trial. Lancet Neurol 2008;7:299-309

31. Hacke W, Albers G, Al-Rawi Y, et al. The Desmoteplase in Acute Ischemic Stroke Trial (DIAS): a phase II MRI-based 9-hour window acute stroke thrombolysis trial with intravenous desmoteplase. Stroke 2005;36:66-73

32. Kimura K, Iguchi Y, Shibazaki K, et al. Recanalization within one hour after intravenous tissue plasminogen activator is associated with favorable outcome in acute stroke patients. Eur Neurol 2010;63:331-36

33. Saqqur M, Uchino K, Demchuk AM, et al. Site of arterial occlusion identified by transcranial Doppler predicts the response to intravenous thrombolysis for stroke. Stroke 2007;38:948-54

34. del Zoppo GJ, Poeck K, Pessin MS, et al. Recombinant tissue plasminogen activator in acute thrombotic and embolic stroke. Ann Neurol 1992;32:78-86

35. Kucinski T, Koch C, Eckert B, et al. Collateral circulation is an independent radiological predictor of outcome after thrombolysis in acute ischaemic stroke. Neuroradiology 2003;45:11-18

36. Christou I, Felberg RA, Demchuk AM, et al. Intravenous tissue plasminogen activator and flow improvement in acute ischemic stroke 
patients with internal carotid artery occlusion. J Neuroimaging 2002;12:119-23

37. Jansen $\mathrm{O}$, von Kummer R, Forsting M, et al. Thrombolytic therapy in acute occlusion of the intracranial internal carotid artery bifurcation. AJNR Am J Neuroradiol 1995;16:1977-86

38. Murphy BD, Fox AJ, Lee DH, et al. Identification of penumbra and infarct in acute ischemic stroke using computed tomography perfusion-derived blood flow and blood volume measurements. Stroke 2006;37:1771-77
39. Janardhan V, Qureshi AI. Mechanisms of ischemic brain injury. Curr Cardiol Rep 2004;6:117-23

40. Miteff F, Levi CR, Bateman GA, et al. The independent predictive utility of computed tomography angiographic collateral status in acute ischaemic stroke. Brain 2009;132:2231-38

41. Christoforidis GA, Mohammad Y, Kehagias D, et al. Angiographic assessment of pial collaterals as a prognostic indicator following intra-arterial thrombolysis for acute ischemic stroke. AJNR Am J Neuroradiol 2005;26:1789-97 